\title{
Targeting Brain-Derived Neurotrophic Factor (BDNF) - An Important Strategy to Alzheimer's Disease
}

\author{
Hemant S. Kanhere ${ }^{1, *}$, Pallavi C. Bansinge ${ }^{1}$, J. Helen Ratna Monica ${ }^{2}$, Sawan K. Rathod ${ }^{1}$
}

${ }^{1}$ Division of Neuroscience, Department of Pharmacology, Smt. Kishoritai Bhoyar College of Pharmacy, New Kamptee, Nagpur - 441 002, MS, India. ${ }^{2}$ Post Graduate and Research Department of Chemistry, The American College, Madurai - 625 002, Tamilnadu, India.

\section{ART IC LE DETA ILS}

Article history:

Received 04 June 2021

Accepted 23 July 2021

Available online 28 September 2021

\section{Keywords:}

BDNF

CREB

Alzheimer's Disease

Neurotrophins

$\beta$-Amyloid

\begin{abstract}
A B S T RAC T
Many theories have been proposed to explain why candidate disease-modifying drugs (DMTs) for Alzheimer's disease (AD) failed. Late initiation of treatments during AD development, inappropriate drug dosages, incorrect selection of main therapeutic targets, and primarily inadequate understanding of the complex pathophysiology of $\mathrm{AD}$ are the most prominent ones. Reduced expression of Brain Derived Neurotrophic Factor (BDNF) is essential in the pathogenesis of Alzheimer's disease. BDNF plays important functions in cell survival and differentiation, neuronal outgrowth and plasticity. It can be a novel target for the treatment of the disease. In Alzheimer's disease, the hippocampus, parietal, entorhinal, and frontal cortex all have the most extreme BDNF deficits. Lower levels of BDNF can be linked to neuronal death, masking any gene-related effects. High BDNF levels have been attributed to a lower risk of dementia and Alzheimer's. Improvements in BDNF levels imparted by exercise, plant based drugs, trkB receptor agonist and BDNF enhancer drug have been proved to enhance cognitive performance. Plant-based products and nutraceuticals can boost BDNF levels. Polyphenols are essential plant compounds with a wide range of therapeutic potentials. Flavonoids like calycosin, genistein, isorhamnetin, and luteolin have been shown to affect the level of BDNF. Curcumin, a compound derived from spice turmeric (curcuma longa), has a variety of biological functions in the brain, including antidepressant properties which also increase BDNF level in the hippocampus. Riluzole is used to treat amyotrophic lateral sclerosis (ALS). In a depression model with chronic corticosteroid intake, riluzole also restores hippocampal BDNF levels. Evidence indicates that BDNF deficiency plays a role in the pathogenesis of Alzheimer's disease. Drugs used to treat Alzheimer's disease have the unintended property of modulating BDNF levels in brain regions specifically involved in the disease's pathophysiology. The discovery of molecules that precisely control BDNF in particular cellular phenotypes could increase the effectiveness of therapy against AD.
\end{abstract}

\section{Introduction}

Daily living activities are fundamental skills required to independently care for oneself and are categorized in basic and instrumental activities of daily living. Activities of daily living evaluation is of very importance in clinical practice to discriminate between healthy individuals and patients with mild cognitive impairment (MCI) or Alzheimer's disease (AD). One of the key clinical features of Alzheimer's disease (AD) is impairment in daily functioning. Patients with mild cognitive impairment (MCI) also commonly have mild problems performing complex tasks. Information and communication technology (ICT), particularly techniques involving imaging and video processing, is of interest in order to improve assessment [1-3]. Alzheimer's disease is an age-related disorder characterized by the dysfunction and death of neurons in brain regions, such as the hippocampus and frontal cortex, involved in learning and memory processes. The neurodegenerative process in $\mathrm{AD}$ is believed to involve mitochondrial alterations, membrane-associated oxidative stress, altered proteolytic processing of the $\beta$-amyloid precursor protein (APP) and accumulation of neurotoxic forms of the amyloid $\beta$-peptide $(A \beta)$ Studies of experimental models relevant to $\mathrm{AD}$ have provided evidence that $\mathrm{A} \beta$ and oxidative stress disrupt neuronal $\mathrm{Ca}^{2+}$, thereby rendering neurons vulnerable to excitotoxicity and the development of cytoskeletal alterations characteristic of dying neurons in $\mathrm{AD}$ [4-7].

Neurodegenerative conditions associated with cognitive decline, including $\mathrm{AD}$ are frequently associated with changes in the number and shape of dendritic spines prior to neuronal death. Neurodegeneration in $\mathrm{AD}$ patients is characterized by changes in neurotransmitter expression, reduced neutrophil numbers, synaptotoxicity, accumulation of $A \beta$-protein ( $\beta$ - Amyloid protein) deposits and large-scale neuronal death and neural atrophy in the final phase of the disease. A number of studies have suggested that $A \beta$ accumulation may contribute to dendritic spine loss. Deficits in memory and other cognitive functions in the initial stages of the disease are associated with changes in the hippocampus and the entorhinal cortex. As many as $80 \%$ of the neurons in the hippocampus may die over the course of $\mathrm{AD}$, and this progressive loss is manifest in the cognitive changes and other symptoms seen in $A D$ patients [8]. $A D$ pathogenesis is expected to be caused but the production and deposition of the $\beta$-Many evidences indicates that the solubility and the quantity of $A \beta$ in different pools may be closely related to disease state.

Brain-derived neurotrophic factor, also known and denoted as BDNF, is a protein that, in humans, is encoded by the BDNF Gene. BDNF is a member of the neurotrophin family of growth factors, which are related to the canonical nerve growth factor. Neurotrophic factors are found in the brain and in the periphery. BDNF itself is important for long-term memory. Although the vast majority of neurons in the mammalian brain are formed prenatally, parts of the adult brain retain the ability to grow new neurons from neural stem cells in a process known as neurogenesis. Brain-derived neurotrophic factor (BDNF) is sometimes denoted as miracle grow for the brain" because several studies have reported that BDNF nourishes neurons like that of the fertilizer nourishes a plant $[9,10]$. It is well-known that BDNF protects brain cells and stimulates growth of their dendrites and axons. In addition, BDNF has been associated with enhancement of learning and cognitive function. Brain derived neurotrophic factor (BDNF) is the most prevalent growth factor in the central nervous system (CNS). It is essential for the development of the CNS and for neuronal plasticity. Because BDNF plays a crucial role in development and plasticity of the brain, it is widely implicated in psychiatric diseases. 
This review provides a summary of clinical and preclinical evidence for the involvement of this ubiquitous growth factor in major depressive disorder, schizophrenia, addiction, Rett syndrome, as well as other psychiatric and neurodevelopmental diseases.

Neurotrophins are proteins that help to stimulate and control neurogenesis, BDNF being one of the most active. Mice born without the ability to make BDNF suffer developmental defects in the brain and sensory nervous system, and usually die soon after birth, suggesting that BDNF plays an important role in normal neural development. Other important neurotrophins structurally related to BDNF include NT-3, NT-4, and NGF. These Nerve Growth Factor (NGF) is the archetype of this family of neurotrophins. BDNF is known to promote neuronal survival, axonal guidance, and activity-dependent synaptic plasticity. Acute increases in brain-derived neurotrophic factor in plasma following physical exercise relates to subsequent learning in older adults. BDNF acts on certain neurons of the central nervous system and the peripheral nervous system, helping to support survival of existing neurons, and encouraging growth and differentiation of new neurons and synapses. In the brain it is active in the hippocampus, cortex and basal forebrain-areas vital to learning, memory and higher thinking. BDNF is also found in the retina, kidneys, prostate, motor neurons, and skeletal muscle, and is also found in saliva [11]

BDNF is made in the endoplasmic reticulum and secreted from densecore vesicles. It binds carboxypeptidase E (CPE), and disruption of this binding has been proposed to cause the loss of sorting BDNF into densecore vesicles. The phenotype for BDNF knockout mice can be severe, including postnatal lethality. Other traits include sensory neuron losses that affect coordination, balance, hearing, taste, and breathing. Knockout mice also exhibit cerebellar abnormalities and an increase in the number of sympathetic neurons. BDNF binds at least two receptors on the surface of cells that are capable of responding to this growth factor. It may also modulate the activity of various neurotransmitter receptors, including the Alpha-7 nicotinic receptor. BDNF has also been shown to interact with the reelin signaling chain. The expression of reelin by Cajal-Retzius cells goes down during development under the influence of BDNF. The latter also decreases reelin expression in neuronal culture [12].

Various studies have shown possible links between BDNF and conditions, such as depression, schizophrenia, obsessive-compulsive disorder, Alzheimer's disease, Huntington's disease, Rett syndrome, and dementia, as well as anorexia nervosa and bulimia nervosa. Increased levels of BDNF can induce a change to an opiate-dependentlike reward state when expressed in the ventral tegmental area in rats. Post mortem analysis has shown lowered levels of BDNF in the brain tissues of people with Alzheimer's disease, although the nature of the connection remains unclear. Studies suggest that neurotrophic factors have a protective role against amyloid beta toxicity [7-13].

\section{BDNF and Alzheimer's Disease}

Alzheimer's Disease and BDNF Reduced BDNF levels have been reported in a variety of pathological disorders, including Huntington's disease (HT), Alzheimer's disease (AD), and Parkinson's disease. "However, the profile of cognitive deficits greatly differs between these pathologies according to the brain regions affected by degeneration" [14]. The hippocampus, parietal, entorhinal, and frontal cortex, in all, have the most severe BDNF deficits in Alzheimer's disease [14]. Alzheimer's disease manifests itself mostly as a loss of declarative memory, with no other neurological processes affected [15]. This function can be attributed to the degenerative profile of the disease, which begins in the hippocampus, parahipocampal cortices, and amygdala rather than the main sensory and motor cortices [16].The BDNF encoding gene has been illustrated as a target for susceptibility to Alzheimer's disease-related depression (AD-D) [17]. There are various studies that support the idea that neurotrophic factors, especially BDNF, are important in the aetiology of AD. When BDNF protein and mRNA levels [14] and proBDNF levels [18] decline comparable to age-matched controls in the post-mortem brain of patients with AD, there are no differences in TrkB levels [19]. Mild Cognitive Impairment (MCI) also showed this decline [20], a potential prodromal phase of $\mathrm{AD}$ [21]. In addition, MCI patients had lower circulating levels of BDNF [22]. BDNF levels are linked to disease incidence and episodic memory output in patients [18], meaning that these reductions are linked to the disease's pathogenesis. Finally, downregulation of BDNF and proBDNF is believed to be an underlying cause of early Alzheimer's disease [18]. Laske et al. [23] discovered that patients in the early stages of Alzheimer's disease had slightly higher sBDNF levels than late-stage patients and age-matched monitors. Since the pathology is characterised by a loss of cell density and dendritic spines, which could influence BDNF levels secondarily, it is difficult to determine a causal connection between

BNDF downregulation and the progression of this neurodegenerative disease. There are also results that indicate a rise in BDNF and TrkB concentrations in the hippocampus as well as the parietal cortex of patients with $\mathrm{AD}[24,25]$. This rise may be linked to compensatory pathways that help with $\beta$-amyloid degradation and repair. Other alternative moderators may also play a role in the variations and heterogeneity observed in these studies. Distinctions in diagnosis criteria, disease stages, sex and education, including the use of therapeutic medications such as acetylcholinesterase inhibitors or psychotropic medicine that are suspected to increase BDNF levels [26] or from other alternative causes outside the CNS such as immune cells [27]. Given that synaptic loss is a far greater predictor of cognitive decline than plaques or tangles, [28] there has been a recent shift in thinking about Alzheimer's disease as a "synaptic pathology" $[29,30]$. A $\beta$ monomers are naturally produced and secreted at firing synapses, and they are not toxic but neuroprotective because they play an important role in synaptic control $[31,32] . A \beta$ monomers are one of several factors that control synaptic activity, and they can activate CREB through the PI3K/AKT pathway, resulting in CREB-regulated transcription and BDNF release that is sustained $[33,34]$. In this way, BDNF can serve as a hub for a variety of synaptic regulators. Self-association of $A \beta$ monomers produces neurotoxic $\beta$-amyloid $(A \beta)$ oligomers in Alzheimer's disease (AD). These oligomers can cause neurotoxicity in a variety of ways [35]. According to Arshavsky [36], the selective weakness of memory-related areas may be the result of complex cellular modifications needed for memory consolidation. Pathogenic $A \beta$-mediated alterations in the levels of neurotrophic factors (NTFs) are a critical occurrence in AD [37]. Since pathogenic A $\beta$ oligomers can't trigger the PI3K/AKT pathway or induce CREB activation, an increase in A $\beta$-oligomer levels can cause CREB activation to be impaired in the brains of Alzheimer's patients and mouse models [38]. Soluble $A \beta$ oligomers have been shown to affect signal transduction pathways important for learning and memory, such as CREB-regulated transcription [39] and NMDA receptor trafficking [40]. As a result, changes in those pathways may play a key role in the disease's aetiology. BDNF levels in AD are affected by $A \beta$ accumulation and may be linked to $A \beta$-induced CREB transcription dysregulation $[39,41]$. Even if BDNF does not affect $A \beta$ accumulation, it can play a key role in modulating $A \beta$ impact on cognitive and structural aspects [42]. By leading to $A \beta$ degradation and preventing tau hyperphosphorylation, BDNF protects against $\mathrm{A} \beta$-mediated toxicity $[43,44]$. BDNF is expressed by microglia and astroglia in the vicinity of the plaque and appears to defend against neuroinflammation, promoting neuronal survival and preventing apoptosis $[27,45]$. $A \beta$, on the other hand, inhibits retrograde axonal transport of BDNF [46] and the conversion of pro-BDNF to mature BDNF in vitro by reducing CREB $[47,48]$. Additionally, at concentrations that do not cause cell death, it impairs the synaptic plasticity mediated by BDNF [49]. This downregulation occurs prior to plaque formation and has been related to memory deficits in AD animal models [50] and MCI patients [18]. Tau, a mediator of $A \beta$-induced toxicity, can downregulate BDNF in vitro and in vivo by itself through transcript IV [51]. As previously reported, several studies have shown that reductions in serum BDNF levels can be observed in people with $\mathrm{MCI}$, so it's tempting to think that BDNF loss is a precursor to synaptic dysfunction. However, some differences between studies with MCI patients caution us to tread carefully when making these assumptions. Nonetheless, these findings indicate that BDNF plays a critical role in the control of $A \beta$-amyloid toxicity, indicating that BDNF dysregulation can play a role in synaptic dysfunction and mnemonic impairment in Alzheimer's disease [84]. Changes in BDNF expression may be an effect of earlier functional modifications in other synaptic related proteins, implying that, although essential to the production of $\mathrm{AD}$, changes in BDNF expression may be an effect of earlier functional modifications in other synaptic related proteins. One of these proteins, in particular, may be $A \beta$, which has a normal physiological role in synaptic plasticity and neuronal survival in the brain in its monomeric form and may potentially play a role in these BDNF changes by controlling BDNF transcription and release [52]. In either case, BDNF's memory and cognition-enhancing properties may be due to its synapse-repair properties. Alterations in the cell microenvironment, such as a loss of trophic reinforcement, which may contribute to a decline in neuronal survival and proliferative activity [53], can play an important role in the pathological degeneration of specific neuronal subpopulations. During this period, increases in BDNF levels trigger age-related hippocampal volume changes and atrophy, which are connected to pathological conditions [54]. In the elderly without symptoms of dementia, there is evidence of $1-2 \%$ annual hippocampal atrophy, while in patients with Alzheimer's disease, this decline is $3-5 \%$ per year. Hippocampal volume is predictive of rapid conversion to dementia in patients with MCI [55], demonstrating its role in the progression of the disease. The majority of studies suggest that the BDNF genotype has no bearing on the likelihood of developing Alzheimer's 
disease [56-58] [but see Fukumoto et al. [59] for proof of results only in women]. However, several studies have shown that Val carriers have a higher risk of Alzheimer's disease [60-63]. Other research has linked Met carriers to higher rates of episodic memory loss and hippocampal atrophy in patients with $\mathrm{MCI}$ [64-66] although not to $A \beta$ accumulation [65]. Despite some discrepancies in the literature, BDNF's role in the development of Alzheimer's disease has been replicated with other BDNF polymorphisms $[67,68]$. The discrepancy between studies may be attributed to variations in BDNF effect at different phases of the illness, with more circulating BDNF in MCI patients and fewer in AD patients $[69,64]$. Lower BDNF levels could be related to neuronal death in Alzheimer's disease, masking any BDNF gene impact. Since the pathological changes caused by the disease become more complex as the severity increases, correlations between the BDNF Val66Met polymorphism and AD should be more evident in preclinical stages, where the disease is almost exclusively manifested by subtle changes in mnemonic efficiency [70]. Neurotrophic factors help to prevent not only neural and synaptic dysfunction, but also cognitive impairment in Alzheimer's patients [70]. Higher levels of sBDNF have been linked to a reduced risk of dementia and Alzheimer's disease in the future [71] as well as slower rates of decline [72]. Similarly, pharmacologically mediated or aerobic exercise-induced improvements in BDNF levels are linked to improved cognitive function and reduced synaptic dysfunction in both humans at risk of developing $\mathrm{AD}$ and animal models of $\mathrm{AD}[73,74]$. These results may be linked to BDNF's ability to prevent neuronal degeneration caused by lesion $[75,76]$. According to this theory, BDNF gene transfer after a lesion partially restored learning ability and synaptic plasticity deficits in an AD model in which BoNTx-induced entorhinal cortex damage was used to simulate AD pathology [77]. "Despite widespread $A \beta$ plaque and tau pathology, neural stem cell transplants or CREB binding protein gene transfers reversed spatial memory deficits in AD mouse models via BDNF $[78,39]$. In analysis, administering BDNF to the entorhinal cortex of amyloid transgenic mice restored neuronal atrophy and synaptic loss, regulated neuronal signalling, and reduced associated mnemonic deficits without influencing amyloid plaque load [42], illustrating that BDNF can exert its protective effect by amyloid-independent mechanisms. Moreover, in lab animals of Alzheimer's disease as well as in preclinical trials, 7,8 -dihydroflavone $(7,8$ DHF), Neotrofin (a hypoxanthine derivative that inhibits neurotrophic factor production), and Neuropep-1 (a BDNF modulating peptide) have been seen to reverse memory deficits [79-81, 84]. In this way, BDNF can play a role in mediating the anti-degenerative effects of exercise and calorie restriction [82]. This emphasises the importance of developing therapeutic approaches to reduce the risk of developing dementia or to delay the development of dementia in MCI patients, a direction that is currently being pursued. Many of these new paths point to behavioural modifications as possible therapies, including antioxidant diets, environmental enrichment, and social activity, as well as physical and cognitive exercise [83].

Fig. 1 shows Alzheimer's disease is connected by the molecular pathways mediated by BDNF. External stimuli that cause epigenetic regulation processes (stress, maternal deprivation, or lack of activity) may result in a decrease in BDNF expression and neuronal activity. Atrophy, neuronal death, and cognitive loss occur as a result, which can lead to Alzheimer's disease. The BDNF/TrkB signalling pathway, which stimulates downstream Akt and ERK signalling, is disrupted in these diseases. As a consequence, such changes disrupt CREB signalling, resulting in BDNF downregulation [85].
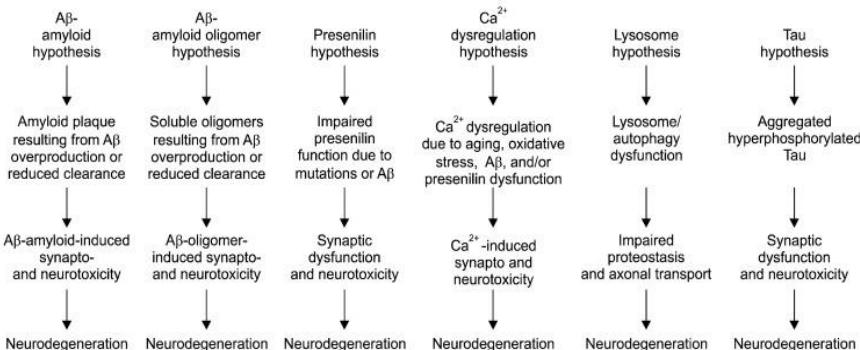

Fig. 1 Pathogenic hypotheses for synaptic and neuronal toxicity in Alzheimer's disease

\section{Targeting BDNF Signalling Pathway by Natural Products}

Clinical trials for the treatment of Alzheimer's disease using viral vector-mediated BDNF gene delivery in the entorhinal cortex area of $A D$ patients are still in the early stages [86, 99]. As a result, using plant-based products and nutraceuticals to boost BDNF levels is a safer option.
Polyphenols are essential plant compounds with a wide range of therapeutic potential, and an increasing body of evidence suggests that they can play a role in neuroprotection [87-90]. Various polyphenolic compounds, such as catechin, chlorogenic acid, luteolin, silybin, calycosin, genistein, hyperforin, and hypericin, have been shown to affect BDNF in several studies. Polyphenols have been shown to increase BDNF levels in the brain by activating the extracellular signal-regulated kinase (ERK) and cyclic adenosine monophosphate (cAMP) response element binding protein (CREB-(a synaptic plasticity associated protein)) cascades, both of which are involved in BDNF gene expression $[84,88,99]$. Polyphenols bind to BDNF TrkB receptors and function as agonists [91]. The natural compounds that may have an impact on BDNF are mentioned below. We also highlight some research on plants and their extracts that have BDNFstimulating properties. The majority of the compounds had an up-or down-regulating effect on BDNF levels in the brain [92, 93] Complementary and complementary medicine may be a lifesaver for people suffering from such illnesses. Green tea, Ginkgo biloba, Quercus robur extracts, curcumin, and other natural products can be helpful to patients suffering from depression by affecting the neuronal system [94]. In a murine model of depression, compounds like ginsenoside Rg1 were found to have neuroprotective and antidepressant-like effects by activating the cAMP response element binding protein (CREB)-BDNF system inside the basolateral amygdala [95]. In rats, curcumin treatment increased BDNF levels and significantly decreased depression-like behaviour [96]. In mouse models of depression, long-term administration of green tea polyphenols increased BDNF levels and decreased adrenocorticotropic hormone and cortisol levels, as well as improved psychological stress-induced cognitive dysfunctions [97,98]. Honokiol, magnolol, spicatoside A, catechin, chlorogenic acid, alpinetin, luteolin, silybin, calycosin, genistein, fisetin, isorhamnetin, fucoxanthin, corallocins, eicosapentaenoic acid, scopoletin, hyperforin, and hypericin are all essential polyphenolic compounds with BDNF-induce. The majority of the compounds have been shown to increase BDNF secretion and expression [99]. These compounds are polyphenolic in nature and extracted from natural resources. These are natural antioxidants, according to most experts. Several of these compounds have also gone through different stages of clinical trials [99]. BDNF levels and results have been shown to be boosted by a variety of botanicals and polyphenols. They achieve these effects through a variety of mechanisms, including increased BDNF gene transcription [100], upregulation of BDNF [101] and TrkB [102], ERK and CREB signalling, and extracellular signal regulated kinase (ERK) and CREB signalling [103]. $\mathrm{Xu}$ et al. suggested oestrogen receptor-mediated upregulation of BDNF expression as a potential mechanism for flavonoids like calycosin, genistein, isorhamnetin, and luteolin in enhancing BDNF levels in astrocytes [104]. Sohrabji et al. [105] confirmed the presence of oestrogen response elements in the BDNF gene and estrogen-mediated upregulation of BDNF transcription, which supports this hypothesis. In astrocytes, the caffeine ester fraction and scutellarin increase BDNF synthesis. According to Chai et al. [106], this impact may be due to increased CREB and Akt phosphorylation. CREB and Akt phosphorylationmediated increased BDNF synthesis has been proposed by Chai et al. as a potential mechanism for BDNF upregulation. 7,8-dihydroxyflavone, on the other hand, has been shown to mimic BDNF behaviour rather than modulating BDNF levels or effects. The agonistic action of 7,8dihydroxyflavone at TrkB receptors in the hippocampal and motor neurons produces a BDNF-like effect $[107,108]$.

\section{Pharmacological Interventions to Up-Regulate BDNF Signalling} Agents that Increase BDNF Levels

The terminal region of the CoA molecule is made up of cysteamine, which is a natural product of cells. Cysteamine, a natural product of cells, makes up the terminal region of the CoA molecule. Cysteine and a similar drug, cystamine, have been shown in studies to have neuroprotective effects in Huntington's disease mice by increasing central BDNF levels [109]. In addition, cystamine or cysteamine injection will raise serum BDNF levels in both wild-type and HD mice. Cytamine or its derivatives may have possible antidepressant therapeutic effects since activation of the BDNF-dependent pathway plays a key role in the mechanism of antidepressant therapeutic action [110]. Deltamethrin, a pyrethroid insecticide, has been shown to improve BDNF gene expression and thus has antidepressant therapeutic potential [111]. A recent study in mice supported this finding, demonstrating that deltamethrin can reduce immobility time in the FST. Deltamethrin's antidepressant-like effects have been blocked by intracerebroventricular injection of a BDNF receptor TrkB inhibitor, indicating that deltamethrin's antidepressant-like effects are mediated by BDNF signalling pathways [112]. Over 100 enzymes involved in the metabolism of carbohydrates, fatty acids, 
proteins, and nucleic acids include zinc as a cofactor. Chronic zinc treatment has been shown to increase the levels of BDNF mRNA in the rat cerebral cortex [113]. In the brain, hydrogen sulphide $\left(\mathrm{H}_{2} \mathrm{~S}\right)$ acts as a neuroprotectant against oxidative stress. In the hippocampus of chronic unpredictable moderate stress rats, $\mathrm{H}_{2} \mathrm{~S}$ has been shown to upregulate the levels of BDNF and p-TrkB protein [114]. Curcumin, a compound derived from curry spice, has a variety of biological functions in the brain, including antidepressant-like properties. Chronic curcumin also increases hippocampal BDNF in a dose-dependent manner [115]. The drug riluzole is used to treat amyotrophic lateral sclerosis (ALS). In a depression model with chronic corticosteroid intake, riluzole also restores hippocampal BDNF levels [116]. Harmine is a $\beta$-carboline alkaloid extracted from Ayahuasca, a psychotropic herb decoction. In the rat hippocampus, repeated administration of harmine raises BDNF protein levels $[117,118]$.

\section{Non-Pharmacological Intervention Lifestyle}

The prospect of interfering with the progression of Alzheimer's disease by basic yet difficult behavioural changes may have consequences for preventing cognitive deterioration associated with the disease [119]. Lazarov et al. [120] have elegantly shown that an enriched environment results in a significant reduction of $\beta$-amyloid deposits in the brain as compared to animals housed in normal conditions. This finding is consistent with previous research that suggests synaptic activity [121], which is increased in an enriched environment, may influence b-amyloid development. While dissecting the various components of environmental manipulations is difficult, we must consider that increased physical activity can play a role. In a transgenic model of Alzheimer's disease, exercise has recently been shown to reduce bamyloid build-up [122]. This finding is in line with an epidemiologic study that found a significantly higher risk of cognitive impairment in inactive patients [123], as well as another study that showed physical activity protects against cognitive decline [124]. Food consumption can change as a result of changes in lifestyle. As a result, the possibility that diet could affect the onset and progression of AD has been considered. Dietary factors in Alzheimer's disease have been thoroughly studied by Luchsinger and Mayeux [125]. They conclude that, while epidemiological studies have looked into the connection between diet and Alzheimer's disease, the findings aren't conclusive. Only a few studies have shown the possible benefit of the micronutrient vitamin $\mathrm{E}$ in the prevention of Alzheimer's disease, [126129] indicating that oxidative stress inhibition could be significant in delaying the development of the disease. Markers of oxidative damage, such as increased lipid peroxidation or increased protein and DNA oxidation, are also affected in the brains of AD patients, according to postmortem studies [130]. While addressing the molecular mechanisms through which the environment may function in animal models of AD is difficult, it is worth noting that BDNF is a common denominator that is associated with the various lifestyle changes discussed above and their effect on cognition. In reality, rats raised in an enriched environment perform better on a spatial memory task and have higher levels of BDNF in the hippocampus and cerebral cortex, according to Ickes et al. [131]. Similarly, Lazarov et al. [120] found that BDNF levels are higher in animal models of Alzheimer's disease with lower levels of bamyloid deposition. These findings suggest that BDNF can play a role in mediating the benefits of enriched conditions in animal models of Alzheimer's disease. Vaynman et al. [132] investigated the molecular basis for the increase in cognition caused by physical activity and discovered that physical exercise improved cognitive functions by upregulating BDNF. BDNF tends to play a role in the recovery of function after traumatic brain injury [133], implying that it plays a critical role in mediating the beneficial effects of voluntary exercise. In terms of diet, only vitamin $\mathrm{E}$ has been shown to be involved in epidemiological research looking at food intake and Alzheimer's disease [126-129]. Vitamin E, interestingly, reversed the decreases in BDNF expression and CREB phosphorylation in the hippocampus caused by a high-fat diet, while also improving cognitive functions [134]. These findings indicate that vitamin E supplementation could be a developing therapeutic choice for diseases characterised by oxidative damage, such as Alzheimer's disease, by enhancing the BDNF and CREB pathways [135].

\section{Discussion}

Reduced BDNF levels have been reported in Huntington's disease, Alzheimer's disease and Parkinson's disease. The hippocampus, parietal, entorhinal, and frontal cortex, in all, have the most severe BDNF deficits. Downregulation of BDNF and proBDNF is believed to be an underlying cause of early Alzheimer's Disease. It is difficult to determine a causal connection between BNDF downregulation and the progression of this https://doi.org/10.30799/jpmr.055.21060103 neurodegenerative disease. $A \beta$ monomers are naturally produced and secreted at firing synapses. They are not toxic but neuroprotective because they play an important role in synaptic control. $A \beta$-mediated alterations in the levels of neurotrophic factors (NTFs) are a critical occurrence in Alzheimer's disease. Soluble $A \beta$ oligomers have been shown to affect signal transduction pathways important for learning and memory. Changes in those pathways may play a key role in the disease's aetiology. BDNF dysregulation can play a role in synaptic dysfunction and mnemonic impairment in Alzheimer's disease. Changes in BDNF expression may be an effect of earlier functional modifications in other proteins. Lower BDNF levels could be related to neuronal death, masking any gene impact. Val carriers have a higher risk of developing Alzheimer's. Other research has linked Met carriers to higher rates of episodic memory loss and hippocampal atrophy in patients with MCI, although not to $A \beta$ accumulation. Despite some discrepancies in the literature, BDNF's role in the development of Alzheimer's Disease has been replicated with other BDNF polymorphisms. High levels of sBDNF have been linked to a reduced risk of dementia and Alzheimer's disease in the future. Exercise-induced improvements in BDNF levels are linked to improved cognitive function. External stimuli that cause epigenetic regulation processes (stress, maternal deprivation, or lack of activity) may result in a decrease in BDNF expression and neuronal activity. This emphasises the importance of developing therapeutic approaches to reduce the risk of developing dementia or to delay the development of dementia in MCI patients. Many of these new paths point to behavioural modifications as possible therapies, including antioxidant diets, environmental enrichment, and social activity, as well as physical and cognitive exercise. Plant-based products and nutraceuticals to boost BDNF levels are a safer option. Polyphenols are essential plant compounds with a wide range of therapeutic potential. Various polyphenolic compounds have been shown to affect BDNF in several studies. Green tea, curcumin, Quercus robur extracts, and other natural products can be helpful to patients suffering from depression. Complementary medicine may be a lifesaver for people suffering from such illnesses. Some research on plants and their extracts that have BDNF-stimulating properties. The majority of compounds had an up-or down-regulating effect on BDNF levels in the brain. BDNF levels and results have been shown to be boosted by a variety of botanicals and polyphenols. Botanicals achieve these effects by increasing BDNF gene transcription and upregulation of signalling pathways. 7,8dihydroxyflavone, on the other hand, mimics BDNF behaviour rather than modulating BDNF levels or effects. CREB and Akt phosphorylationmediated BDNF synthesis has been proposed by Chai et al. as a potential mechanism for BDNF upregulation. Cysteine and a similar drug, cystamine, have been shown in studies to have neuroprotective effects in Huntington's disease mice by increasing central BDNF levels. Hydrogen sulphide $\left(\mathrm{H}_{2} \mathrm{~S}\right)$ acts as a neuroprotectant against oxidative stress. Curcumin, a compound derived from curry spice, has a variety of biological functions in the brain, including antidepressant-like properties. Wang et al created the AS86 TrkB agonist antibody, which improves neurite outgrowth and cell survival by directly activating TrkB and its downstream signalling in an in vitro model. Exercise has been shown to reduce bamyloid build-up in a transgenic model of Alzheimer's disease. The possibility that diet could affect the onset and progression of AD has been considered. BDNF levels are higher in animal models of Alzheimer's disease with lower levels of $\beta$-amyloid deposition. Vaynman et al. [132] investigated the molecular basis for the increase in cognition caused by physical activity. Vitamin E supplementation could be a developing therapeutic choice for diseases characterised by oxidative damage, such as Alzheimer's, by enhancing the BDNF and CREB pathways.

\section{Conclusion}

Evidence indicates that BDNF deficiency plays a role in the pathogenesis of Alzheimer's disease. Drugs used to treat Alzheimer's disease have the unintended property of modulating BDNF levels in brain regions specifically involved in the disease's pathophysiology. The ability to finetune BDNF expression in specific brain sites can enable a general refinement of strategies to combat this devastating disorder. The discovery of molecules that precisely control BDNF in particular cellular phenotypes could increase the effectiveness of therapy against $\mathrm{AD}$.

\section{References}

[1] J.M. Long, D.M. Holtzman, Alzheimer disease: an update on pathobiology and treatment strategies, Cell 179(2) (2019) 312-339.

[2] R.J. Bateman, T.L. Benzinger, S. Berry, D.B. Clifford, C. Duggan, A.M. Fagan, et al, The DIAN-TU Next Generation Alzheimer's prevention trial: adaptive design and disease progression model, Alzheimers Dement 13 (1) (2017) 8-19. 
[3] L. Vermunt, S.A. Sikkes, A. Van Den Hout, R. Handels, I. Bos, et al, Duration of preclinical, prodromal, and dementia stages of Alzheimer's disease in relation to age, sex, and APOE genotype, Alzheimers Dement 15(7) (2019) 888-898.

[4] C. Reitz, C. Brayne, R. Mayeux, Epidemiology of Alzheimer disease, Nat. Rev. Neurol. 7(3) (2011) 137-152.

[5] Alzheimer's Association, Alzheimer's disease facts and figures, Alzheimers Dement 6(2) (2010) 158-194.

[6] Alzheimer's Association, Alzheimer's disease facts and figures, Alzheimers Dement 13(4) (2017) 325-373.

[7] C. Takizawa, P.L. Thompson, A. van Walsem, C. Faure, W.C. Maier, Epidemiological and economic burden of Alzheimer's disease: a systematic literature review of data across Europe and the United States of America, J. Alzheimers Dis. 43(4) (2015) 1271-1284.

[8] S. Salloway, S. Correia, Alzheimer disease: time to improve its diagnosis and treatment, Cleve Clin. J. Med. 76(1) (2009) 49-58.

[9] J.E. Galvin, C.H. Sadowsky, Practical guidelines for the recognition and diagnosis of dementia, J. Am. Board Fam. Med. 25(3) (2012) 367-382.

[10] D.A. Casey, D. Antimisiaris, J. O'Brien, Drugs for Alzheimer's disease: are they effective?. Pharm. Therap. 35(4) (2010) 208-211.

[11] A. Qaseem, V. Snow, J.T. Cross Jr, M.A. Forciea, R. Hopkins Jr, et al, Current pharmacologic treatment of dementia: a clinical practice guideline from the American College of Physicians and the American Academy of Family Physicians, Annal. Int. Med. 148(5) (2008) 370-378.

[12] S.R. Tanqueiro, R.M. Ramalho, T.M. Rodrigues, L.V. Lopes, A.M. Sebastião, et al., Inhibition of NMDA receptors prevents the loss of BDNF function induced by amyloid $\beta$, Front. Pharmacol. 9 (2018) 237.

[13] S.S. Jiao, L.L. Shen, C. Zhu, X.L. Bu, Y.H. Liu, et al, Brain-derived neurotrophic factor protects against tau-related neurodegeneration of Alzheimer's disease, Transl. Psychiatry 6(10) (2016) e907.

[14] C. Hock, K. Heese, C. Hulette, C. Rosenberg, U. Otten, et al, Region-specific neurotrophin imbalances in Alzheimer disease: decreased levels of brainderived neurotrophic factor and increased levels of nerve growth factor in hippocampus and cortical areas, Arch. Neurol. 57(6) (2000) 846-851.

[15] D.M. Walsh, D.J. Selkoe, Deciphering the molecular basis of memory failure in Alzheimer's disease, Neuron 44(1) (2004) 181-193.

[16] D.J. Selkoe, Alzheimer's disease: genes, proteins, and therapy, Physiol. Rev. 81 (2001) 741-766

[17] B. Borroni, M. Grassi, S. Archetti, C. Costanzi, M. Bianchi, L. Caimi, BDNF genetic variations increase the risk of Alzheimer's disease-related depression, J. Alzheimers Dis. 18(4) (2009) 867-875.

[18] S. Peng, J. Wuu, E.J. Mufson, M. Fahnestock, Precursor form of brain-derived neurotrophic factor and mature brain-derived neurotrophic factor are decreased in the pre-clinical stages of Alzheimer's disease, J. Neurochem. 93(6) (2005) 1412-1421.

[19] E. Savaskan, F. Müller-Spahn, G. Olivieri, S. Bruttel, U. Otten, et al, Receptor immunoreactivities in parietal cortex and cerebellum in Alzheimer's disease, Eur. Neurol. 44(3) (2000) 172-180.

[20] H. Shimada, H. Makizako, D. Yoshida, K. Tsutsumimoto, Y. Anan, et al, A large, cross-sectional observational study of serum BDNF, cognitive function, and mild cognitive impairment in the elderly, Front Aging Neurosci. 6 (2014) 69.

[21] C. Flicker, S.H. Ferris, B. Reisberg, Mild cognitive impairment in the elderly: predictors of dementia, Neurolo. 41(7) (1991) 1006-1009.

[22] O.V. Forlenza, B.S. Diniz, A.L. Teixeira, E.B. Ojopi, L.L. Talib, V.A. Mendonça, et al, Effect of brain-derived neurotrophic factor Val66Met polymorphism and serum levels on the progression of mild cognitive impairment, World J. Biol. Psychiatry 11(6) (2010) 774-780.

[23] C. Laske, E. Stransky, T. Leyhe, G.W. Eschweiler, A. Wittorf, et al, Stagedependent BDNF serum concentrations in Alzheimer's disease, J. Neural Transm. 113(9) (2006) 1217-1224

[24] N. Durany, T. Michel, J. Kurt, F.F. Cruz-Sánchez, J. Cervós-Navarro, P. Riederer, et al, Brain-derived neurotrophic factor and neurotrophin-3 levels in Alzheimer's disease brains, Int. J. Dev. Neurosci. 18(8) (2000) 807-813.

[25] S.E. O'Bryant, V. Hobson, J.R. Hall, S.C. Waring, W. Chan, et al, Brain-derived neurotrophic factor levels in Alzheimer's disease, J. Alzheimers Dis. 17(2) (2009) 337-341.

[26] T. Leyhe, E. Stransky, G.W. Eschweiler, G. Buchkremer, C. Laske, et al, Increase of BDNF serum concentration during donepezil treatment of patients with early Alzheimer's disease, Eur. Arch. Psychiatry Clin. Neurosci. 258(2) (2008) 124-128.

[27] M. Kerschensteiner, E. Gallmeier, L. Behrens, V.V. Leal, T. Misgeld, et al, Activated human $\mathrm{T}$ cells, B cells, and monocytes produce brain-derived neurotrophic factor in vitro and in inflammatory brain lesions: a neuroprotective role of inflammation?, J. Exp. Med. 189(5) (1999) 865-870.

[28] R.D. Terry, E. Masliah, D.P. Salmon, N. Butters, R. DeTeresa, et al, Physical basis of cognitive alterations in Alzheimer's disease: synapse loss is the major correlate of cognitive impairment, Ann. Neurol. 30(4) (1991) 572-580.

[29] C.F. Lippa, J.E. Hamos, D. Pulaski-Salo, L.J. DeGennaro, D.A. Drachman, et al, Alzheimer's disease and aging: effects on perforant pathway perikarya and synapses, Neurobiol. Aging. 13(3) (1992) 405-411.

[30] J.M. Heffernan, S.L. Eastwood, Z. Nagy, M.W. Sanders, B. McDonald, P.J. Harrison, et al, Temporal cortex synaptophysin mRNA is reduced in Alzheimer's disease and is negatively correlated with the severity of dementia, Exp Neurol. 150(2) (1998) 235-239.

[31] M.L. Giuffrida, F. Caraci, B. Pignataro, S. Cataldo, P. De Bona, et al, $\beta$-amyloid monomers are neuroprotective, J. Neurosci. 29(34) (2009) 10582-10607.

[32] E. Abramov, I. Dolev, H. Fogel, G.D. Ciccotosto, E. Ruff, I. Slutsky, et al, Amyloid$\beta$ as a positive endogenous regulator of release probability at hippocampal synapses, Nature Neurosci. 12(12) (2009) 1567-1576.

[33] M.L. Giuffrida, A. Copani, E. Rizzarelli, A promising connection between BDNF and Alzheimer's disease, Aging (Albany NY) 10(8) (2018) 1791-1792.
[34] S. Zimbone, I. Monaco, F. Gianì, G. Pandini, A.G. Copani, et al, Amyloid Beta monomers regulate cyclic adenosine monophosphate response element binding protein functions by activating type-1 insulin-like growth factor receptors in neuronal cells, Aging Cell 17(1) (2018) e12684.

[35] J. Pearson-Leary, E.C. McNay, Intrahippocampal administration of amyloid- $\beta 1$ 42 oligomers acutely impairs spatial working memory, insulin signaling, and hippocampal metabolism, J. Alzheimers Dis. 30(2) (2012) 413-422.

[36] Y.I. Arshavsky, Alzheimer's disease, brain immune privilege and memory: a hypothesis, J. Neural Transm. 113(11) (2006) 1697-1707.

[37] J. Budni, T. Bellettini-Santos, F. Mina, M.L. Garcez, A.I. Zugno, et al, The involvement of BDNF, NGF and GDNF in aging and Alzheimer's disease, Aging Dis. 6(5) (2015) 331-341.

[38] N. Bartolotti, L. Segura, O. Lazarov, Diminished CRE-induced plasticity is linked to memory deficits in familial Alzheimer's disease mice, J. Alzheimers Dis. 50(2) (2016) 477-489.

[39] A. Caccamo, M.A. Maldonado, A.F. Bokov, S. Majumder, S. Oddo, et al, CBP gene transfer increases BDNF levels and ameliorates learning and memory deficits in a mouse model of Alzheimer's disease, Proc. Nat. Acad. Sci. 107(52) (2010) 22687-22692.

[40] E.M. Snyder, Y. Nong, C.G. Almeida, S. Paul, T. Moran, et al, Regulation of NMDA receptor trafficking by amyloid- $\beta$, Nat. Neurosci. 8(8) (2005) 1051-1058.

[41] S. Pugazhenthi, M. Wang, S. Pham, C.I. Sze, C.B. Eckman, et al, Downregulation of CREB expression in Alzheimer's brain and in A $\beta$-treated rat hippocampal neurons, Mol. Neurodegen. 6(1) (2011) 1-6.

[42] A.H. Nagahara, D.A. Merrill, G. Coppola, S. Tsukada, B.E. Schroeder, et al, Neuroprotective effects of brain-derived neurotrophic factor in rodent and primate models of Alzheimer's disease, Nature Med. 15(3) (2009) 331-337.

[43] E. Elliott, R. Atlas, A. Lange, I. Ginzburg, Brain-derived neurotrophic factor induces a rapid dephosphorylation of tau protein through a PI-3Kinase signalling mechanism, Eur. J. Neurosci. 22(5) (2005) 1081-1089.

[44] L. Tapia-Arancibia, E. Aliaga, M. Silhol, S. Arancibia, New insights into brain BDNF function in normal aging and Alzheimer disease, Brain Res Rev. 59(1) (2008) 201-220.

[45] O. Lindvall, Z. Kokaia, J. Bengzon, E. Elme, M. Kokaia, et al, Neurotrophins and brain insults, Trends Neurosci. 17(11) (1994) 490-496.

[46] W.W. Poon, M. Blurton-Jones, C.H. Tu, L.M. Feinberg, M.A. Chabrier, et al, $\beta$ Amyloid impairs axonal BDNF retrograde trafficking, Neurobiol. Aging 32(5) (2011) 821-833.

[47] E. Rosa, M. Fahnestock, CREB expression mediates amyloid beta-induced basal BDNF downregulation, Neurobiol. Aging 36 (2015) 2406-2413.

[48] Z. Zheng, B. Sabirzhanov, J. Keifer, Oligomericamyloid-\{beta\} inhibits the proteolytic conversion of brain-derived neurotrophic factor (BDNF), AMPA receptor trafficking, and classical conditioning, J. Biol. Chem. 285 (2010) 34708-34717.

[49] D.C. Wang, S.S. Chen, Y.C. Lee, T.J. Chen, Amyloid- $\beta$ at sublethal level impairs BDNF-induced arc expression in cortical neurons, Neurosci. Lett. 398(1-2) (2006) 78-82.

[50] B.M. Francis, J. Yang, E. Hajderi, M.E. Brown, B. Michalski, et al, Reduced tissue levels of noradrenaline are associated with behavioral phenotypes of the TgCRND8 mouse model of Alzheimer's disease, Neuropsychopharm. 37(8) (2012) 1934-1944.

[51] E. Rosa, S. Mahendram, Y.D Ke, L.M. Ittner, S.D. Ginsberg, et al, Tau downregulates BDNF expression in animal and cellular models of Alzheimer's disease, Neurobiol. Aging. 48 (2016) 135-142.

[52] M.S. Parihar, G.J. Brewer, Amyloid- $\beta$ as a modulator of synaptic plasticity, J. Alzheimers Dis. 22(3) (2010) 741-763.

[53] E. Drapeau, D. Nora Abrous, Stem cell review series: Role of neurogenesis in age-related memory disorders, Aging cell 7(4) (2008) 569-589.

[54] K.I. Erickson, D.L. Miller, K.A. Roecklein, The aging hippocampus: interactions between exercise, depression, and BDNF, Neuroscientist 18(1) (2012) 82-97.

[55] C.R. Jack, R.C. Petersen, Y. Xu, P.C. O'Brien, G.E. Smith, R.J. Ivnik, et al, Rate of medial temporal lobe atrophy in typical aging and Alzheimer's disease, Neurolo. 51(4) (1998) 993-999.

[56] O. Combarros, J. Infante, J. Llorca, J. Berciano, Polymorphism at codon 66 of the brain-derived neurotrophic factor gene is not associated with sporadic Alzheimer's disease, Dement. Geriatr. Cogn. Disord. 18(1) (2004) 55-58.

[57] A.L. Nishimura, J.R. Oliveira, M. Mitne-Neto, C. Guindalini, R. Nitrini, et al, Lack of association between the brain-derived neurotrophin factor (C-270T) polymorphism and late-onset Alzheimer's disease (LOAD) in Brazilian patients, J. Mol. Neurosci. 22(3) (2004) 257-260.

[58] Y. Li, C. Rowland, K. Tacey, J. Catanese, J. Sninsky, et al, The BDNF Val66Met polymorphism is not associated with late onset Alzheimer's disease in three case-control samples, Mol. Psychiatry 10(9) (2005) 809-810.

[59] N. Fukumoto, T. Fujii, O. Combarros, M.I. Kamboh, S.J. Tsai, et al, Sexually dimorphic effect of the Val66Met polymorphism of BDNF on susceptibility to Alzheimer's disease: New data and meta-analysis, Am. J. Med. Genet. B Neuropsychiatr. Genet. 153B(1) (2010) 235-242.

[60] J.C. Lambert, B. Grenier-Boley, D. Harold, D. Zelenika, V. Chouraki, et al, Genome-wide haplotype association study identifies the FRMD4A gene as a risk locus for Alzheimer's disease, Mol. Psychiatry 18(4) (2013) 461-470.

[61] M. Ventriglia, L.B. Chiavetto, L. Benussi, G. Binetti, O. Zanetti, et al, Association between the BDNF $196 \mathrm{~A} / \mathrm{G}$ polymorphism and sporadic Alzheimer's disease, Mol. Psychiatry 7(2) (2002) 136-147.

[62] S. Matsushita, H. Arai, T. Matsui, T. Yuzuriha, K. Urakami, et al, Brain-derived neurotrophic factor gene polymorphisms and Alzheimer's disease, J. Neural Transm. 112(5) (2005) 703-710.

[63] A.N. Voineskos, J.P. Lerch, D. Felsky, S. Shaikh, T.K. Rajji, et al, The brain-derived neurotrophic factor Val66Met polymorphism and prediction of neural risk for Alzheimer disease, Arch. Gen. Psychiatry 68(2) (2011) 198-206. 
[64] O.V. Forlenza, B.S. Diniz, A.L. Teixeira, E.B. Ojopi, L.L. Talib, et al, Effect of brainderived neurotrophic factor Val66Met polymorphism and serum levels on the progression of mild cognitive impairment, World J. Biol. Psychiatry 11(6) (2010) 774-780.

[65] Y.Y. Lim, V.L. Villemagne, S.M. Laws, D. Ames, R.H. Pietrzak, et al, BDNF Val66Met, Abeta amyloid, and cognitive decline in preclinical Alzheimer's disease, Neurobiol. Aging 34 (2013) 2457-2464.

[66] Y.Y. Lim, J. Hassenstab, C. Cruchaga, A. Goate, A.M. Fagan, et al, BDNF Val66 Met moderates memory impairment, hippocampal function and tau in preclinical autosomal dominant Alzheimer's disease, Brain 139(10) (2016) 2766-2777.

[67] H. Kunugi, A. Ueki, M. Otsuka, K. Isse, H. Hirasawa, et al, A novel polymorphism of the brain-derived neurotrophic factor (BDNF) gene associated with lateonset Alzheimer's disease, Mol. Psychiatry 6(1) (2001) 83-66.

[68] M. Riemenschneider, S. Schwarz, S. Wagenpfeil, J. Diehl , U. Müller, et al, A polymorphism of the brain-derived neurotrophic factor (BDNF) is associated with Alzheimer's disease in patients lacking the Apolipoprotein E $\varepsilon 4$ allele, Mol. Psychiatry 7(7) (2002) 782-785

[69] H. Yu, Z.J. Zhang, Y.M. Shi, F. Bai, Y. Qian, et al, Cognitive function, serum BDNF levels and BDNF gene Val66Met polymorphism in amnestic mild cognitive impairment, J. Central South Univ. 33(4) (2008) 321-325.

[70] M. Fahnestock, Brain-derived neurotrophic factor: the link between amyloid- $\beta$ and memory loss, Future Neurol. 6(5) (2011) 627-639.

[71] G. Weinstein, A.S. Beiser, S.H. Choi, S.R. Preis, T.C. Chen, et al, Serum brainderived neurotrophic factor and the risk for dementia: the Framingham Heart Study, JAMA Neurol. 71(1) (2014) 55-61.

[72] C. Laske, K. Stellos, N. Hoffmann, E. Stransky, G. Straten, et al, Higher BDNF serum levels predict slower cognitive decline in Alzheimer's disease patients, Int. J. Neuropsychopharm.14(3) (2011) 399-404.

[73] P.A. Barker, Whither proBDNF?, Nat. Neurosci. 12(2) (2009) 105-106.

[74] K.A. Intlekofer, C.W. Cotman, Exercise counteracts declining hippocampal function in aging and Alzheimer's disease, Neurobiol. Dis. 57 (2013) 47-55.

[75] J.K. Morse, S.J. Wiegand, K. Anderson, Y. You, N. Cai, et al, Brain-derived neurotrophic factor (BDNF) prevents the degeneration of medial septal cholinergic neurons following fimbria transection, J. Behav. Neurosci. 13(10) (1993) 4146-4156.

[76] I. Kiprianova, T.M. Freiman, S. Desiderato, S. Schwab, R. Galmbacher, et al, Brain-derived neurotrophic factor prevents neuronal death and glial activation after global ischemia in the rat, J. Behav. Neurosci. Res. 56(1) (1999) 21-27.

[77] S. Ando, S. Kobayashi, H. Waki, K. Kon, F. Fukui, et al, Animal model of dementia induced by entorhinal synaptic damage and partial restoration of cognitive deficits by BDNF and carnitine, J. Neurosci. Res. 70(3) (2002) 519-527.

[78] M. Blurton-Jones, M. Kitazawa, H. Martinez-Coria, N.A. Castello, F.J. Müller, et al, Neural stem cells improve cognition via BDNF in a transgenic model of Alzheimer disease, Proc. National Acad. Sci. 106(32) (2009) 13594-13599.

[79] A.J. Glasky, C.L. Melchior, B. Pirzadeh, N. Heydari, R.F. Ritzmann, et al, Effect of AIT-082, a purine analog, on working memory in normal and aged mice, Pharmacol. Biochem. Behav. 47(2) (1994) 325-329.

[80] L. Devi, M. Ohno, 7, 8-Dihydroxyflavone, a small-molecule TrkB agonist, reverses memory deficits and BACE1 elevation in a mouse model of Alzheimer's disease, Neuropsychopharmacol. 37(2) (2012) 434-444

[81] M.K. Shin, H.G. Kim, S.H. Baek, W.R. Jung, D.I. Park, et al, Neuropep-1 ameliorates learning and memory deficits in an Alzheimer's disease mouse model, increases brain-derived neurotrophic factor expression in the brain, and causes reduction of amyloid beta plaques, Neurobiol. Aging 35(5) (2014) 990-1001.

[82] S. Vaynman, Z. Ying, F. Gomez-Pinilla, Hippocampal BDNF mediates the efficacy of exercise on synaptic plasticity and cognition, Eur. J. Neurosci. 20(10) (2004) $2580-2590$

[83] M. Fahnestock, M. Marchese, E. Head, V. Pop, B. Michalski, et al, BDNF increases with behavioral enrichment and an antioxidant diet in the aged dog, Neurobiol. Aging 33(3) (2012) 546-554.

[84] M. Miranda, J.F. Morici, M.B. Zanoni, P. Bekinschtein, Brain-derived neurotrophic factor: a key molecule for memory in the healthy and the pathological brain, Front Cell Neurosci. 13 (2019) 363.

[85] L. Colucci-D'Amato, L. Speranza, F. Volpicelli, Neurotrophic Factor BDNF, Physiological functions and therapeutic potential in depression, Neurodegen. Brain Cancer, Int. J. Mol. Sci. 21(20) (2020) 7777

[86] A.H. Nagahara, M.H. Tuszynski, Potential therapeutic uses of BDNF in neurological and psychiatric disorders, Nat. Rev. Drug Discov. 10 (2011) 209219

[87] K.B. Pandey, S.I. Rizvi, Plant polyphenols as dietary antioxidants in human health and disease, Oxid. Med. Cell. Longev 2 (2009) 270-278.

[88] D. Vauzour, Polyphenols and brain health, Oilseeds Fats Crops Lipids 24 (2017) A202.

[89] L. Letenneur, C. Proust-Lima, A. Le Gouge, J.F. Dartigues, P. Barberger-Gateau, et al, Flavonoid intake and cognitive decline over a 10 -year period, Am. J. Epidemiol. 165 (2007) 1364-1371.

[90] K. Ono, M.M. Condron, L. Ho, J. Wang, W. Zhao, et al, Effects of grape seedderived polyphenols on amyloid beta-protein self-assembly and cytotoxicity, J. Biol. Chem. 283 (2008) 32176-32187.

[91] F. Moosavi, R. Hosseini, L. Saso, O. Firuzi, Modulation of neurotrophic signaling pathways by polyphenols, Drug Des. Devel. Ther. 10 (2016) 23-42.

[92] J. Trebatická, Z. Duračková, Psychiatric disorders and polyphenols: Can they be helpful in therapy?, Oxid. Med. Cell. Longev. 2015 (2015) 248529.

[93] T. Sharma, L.S. Guski, N. Freund, P.C. Gøtzsche, Suicidality and aggression during antidepressant treatment: systematic review and meta-analyses based on clinical study reports, Brit. Med. Jour. 352 (2016) i65.

[94] D. Tewari, H.K. Pandey, A.N. Sah, H. Meena, V. Chander, Phytochemical, Antioxidant and antidepressant evaluation of Ocimum basilicum, O. tenuiflorum, O. kilimandscharicum grown in India, J. Biol. Act. Prod. Nat. 5(2) (2015) 120-131.

[95] H. Yu, C. Fan, L. Yang, S. Yu, Q. Song, et al, Ginsenoside Rg1 prevents chronic stress-induced depression-like behaviors and neuronal structural plasticity in rats, Cell. Physiol. Biochem. 48 (2018) 2470-2482.

[96] Z. Huang, X.M. Zhong, Z.Y. Li, C.R. Feng, A.J. Pan, Q.Q .Mao, et al, Curcumin reverses corticosterone-induced depressive-like behavior and decrease in brain BDNF levels in rats, Neurosci. Lett. 493 (2011) 145-148.

[97] W.Q. Chen, X.L. Zhao, Y. Hou, S.T. Li, Y. Hong, et al, Protective effects of green tea polyphenols on cognitive impairments induced by psychological stress in rats, Behav. Brain Res. 202 (2009) 71-76.

[98] W.L. Zhu, H.S. Shi, Y.M. Wei, S.J. Wang, C.Y. Sun, et al, Green tea polyphenols produce antidepressant-like effects in adult mice, Pharmacol. Res. 65 (2012) 74-80.

[99] S. Bawari, D. Tewari, S. Argüelles, A.N. Sah, S.F. Nabavi, et al, Targeting BDNF signaling by natural products: novel synaptic repair therapeutics for neurodegeneration and behavior disorders, Pharmacol. Res. 148 (2019) 104458.

[100] K. Morita, N. Nishibori, R. Kishibuchi, M. Itoh, Y. Horie, et al, Fermented brown rice extract stimulates BDNF gene transcription in C6 glioma cells: Possible connection with HO-1 expression, J. Diet. Suppl. 14 (2017) 214-228.

[101] S.M. Ahn, Y.R. Kim, H.N. Kim, H.K. Shin, B.T. Choi, et al, Beneficial effects of polygonum multiflorum on hippocampal neuronal cells and mouse focal cerebral ischemia, Am. J. Chin. Med 43 (2015) 637-651.

[102] S.H. Lee, I.G. Ko, S.E. Kim, L. Hwang, J.J. Jin, H.H. Choi, C.J. Kim, et al, Aqueous extract of cordyceps alleviates cerebral ischemia-induced short-term memory impairment in gerbils, J. Exerc. Rehabil. 12 (2016) 69-78.

103] J.S. Bae, M. Han, H.S. Shin, D.H. Shon, S.T. Lee, et al, Lycopersicon esculentum extract enhances cognitive function and hippocampal neurogenesis in aged mice, Nutrients 8(11) (2016) 679.

[104] S.L. Xu, C.W.C. Bi, R.C.Y. Choi, K.Y. Zhu, A. Miernisha, et al, Flavonoids induce the synthesis and secretion of neurotrophic factors in cultured rat astrocytes: A signaling response mediated by estrogen receptor, Evid. Based. Complement. Alternat. Med. 2013 (2013) 127075

[105] F. Sohrabji, R.C. Miranda, C.D. Toran-Allerand, Identification of a putative estrogen response element in the gene encoding brain-derived neurotrophic factor, Proc. Natl. Acad. Sci. USA 92 (1995) 11110-11114.

[106] L. Chai, H. Guo, H. Li, S. Wang, Y. Wang, et al, Scutellarin and caffeic acid ester fraction, active components of dengzhanxixin injection, upregulate neurotrophins synthesis and release in hypoxia/reoxygenation rat astrocytes, Ethnopharmacol. 150 (2013) 100-107.

[107] S.W. Jang, X. Liu, M. Yepes, K.R. Shepherd, G.W. Miller, et al, Selective TrkB agonist with potent neurotrophic activities by 7,8-dihydroxyflavone, Proc. Natl. Acad. Sci. USA 107 (2010) 2687-2692.

[108] T. Tsai, A. Klausmeyer, R. Conrad, C. Gottschling, M. Leo, et al, 7,8 Dihydroxyflavone leads to survival of cultured embryonic motoneurons by activating intracellular signaling pathways, Mol. Cell. Neurosci. 56 (2013) 1828.

[109] S.J. Tsai, Targeting brain-derived neurotrophic factor (BDNF) signaling pathway as a treatment strategy for depression, Taiwan J. Psych. 32 (2018) 103-113.

[110] S.J. Tsai, Cysteamine-related agents could be potential antidepressants through increasing central BDNF levels, Med. Hypotheses 67 (2006) 1185-1188.

[111] S.J. Tsai, Deltamethrin, a pyrethroid insecticide, could be a potential antidepressant agent, Med. Hypotheses 66 (2006) 605-608.

[112] I. Takasaki, K. Oose, Y. Otaki, Type II pyrethroid deltamethrin produces antidepressant-like effects in mice, Behav. Brain Res. 257 (2013) 182-188.

[113] G. Nowak, B. Legutko, B. Szewczyk, M. Papp, M. Sanak, et al, Zinc treatment induces cortical brain-derived neurotrophic factor gene expression, Eur. J. Pharmacol. 492 (2004) 57-59.

[114] M. Hu, W. Zou, C.Y. Wang, Hydrogen sulfide protects against chronic unpredictable mild stress induced oxidative stress in hippocampus by upregulation of BDNF-TrkB pathway, Oxid. Med. Cell Longev. 2016 (2016) 2153745.

115] S. Hayley, D. Litteljohn, Neuroplasticity and the next wave of antidepressant strategies, Front Cell Neurosci. 7 (2013) 218.

[116] S.L. Gourley, J.W. Espitia, G. Sanacora, J.R. Taylor, Antidepressant-like properties of oral riluzole and utility of incentive disengagement models of depression in mice, Psychopharmacol. (Berl.) 219 (2012) 805-814.

[117] J.J. Fortunato, G.Z. Réus, T.R. Kirsch, Chronic administration of harmine elicits antidepressant-like effects and increases BDNF levels in rat hippocampus, J. Neural Transm. (Vienna) 117 (2010) 1131-1137.

[118] S. Wang, H. Yao, Y. Xu, R. Hao, W. Zhang, et al, Therapeutic potential of a TrkB agonistic antibody for Alzheimer's disease, Theranostics 10(15) (2010) 6854 6874

119] A.I. Faden, S.M. Knoblach, V.A. Movsesyan, I. Cernak, Novel small peptides with neuroprotective and nootropic properties, J. Alzheimers Dis. 6 (6 Suppl) (2004) S93-S97.

[120] O. Lazarov, J. Robinson, Y.P. Tang, I.S. Hairston, Z. Korade-Mirnics, V.M. Lee, et al, Environmental enrichment reduces Abeta levels and amyloid deposition in transgenic mice, Cell 120 (2005)701-713.

[121] F. Kamenetz, T. Tomita, H. Hsieh, G. Seabrook, D. Borchelt, et al, APP processing and synaptic function, Neuron 37 (2003) 925-937.

[122] P.A. Adlard, V.M. Perreau, V. Pop, C.W. Cotman, Voluntary exercise decrease amyloid load in a transgenic model of Alzheimer's disease, J. Neurosci. 25 (2005) 4217-4221.

[123] R.P. Friedland, T. Fritsch, K.A. Smyth, E. Koss, A.J. Lerner, et al, Patients with Alzheimer's disease have reduced activities in midlife compared with healthy control-group members, Proc. Natl. Acad. Sci. USA 98 (2001) 3440-3445. 
[124] D. Laurin, R. Verreault, J. Lindsay, K. MacPherson, K. Rockwood, Physical activity and risk of cognitive impairment and dementia in elderly persons, Arch. Neurol. 58 (2001) 498-504

[125] J.A. Luchsinger, R. Mayeux, Dietary factors and Alzheimer's disease, Lancet Neurol. 3 (2004) 579-587.

[126] M.C. Morris, L.A. Beckett, P.A. Scherr, L.E. Hebert, D.A. Bennett, et al, Vitamin E and vitamin $C$ supplement use and risk of incident Alzheimer disease, Alzheimer Dis. Assoc. Disord. 12 (1998) 121-126.

[127] M.C. Morris, D.A. Evans, J.L. Bienias, C.C. Tangney, D.A. Bennett, et al, Dietary intake of antioxidant nutrients and the risk of incident Alzheimer disease in a biracial community study, J. Am. Med. Assoc. 287 (2002) 3230-3237.

[128] M.C. Morris, D.A. Evans, J.L. Bienias, C.C. Tangney, R.S. Wilson, et al, Vitamin E and cognitive decline in older persons, Arch. Neurol. 59 (2002) 1125-1132.

[129] M.J. Engelhart, M.I. Geerlings, A. Ruitenberg, J.C. Van Swieten, A. Hofman, et al, Dietary intake of antioxidants and risk of Alzheimer disease, J. Am. Med. Assoc. 287 (2002) 3223-3229.
[130] W.R. Markesbery, J.M. Carney, Oxidative alterations in Alzheimer's disease, Brain Pathol. 9 (1999) 133-146.

[131] B.R. Ickes, T.M. Pham, L.A. Sanders, D.S. Albeck , A.H. Mohammed, et al, Longterm environmental enrichment leads to regional increases in neurotrophin levels in rat brain, Exp. Neurol. 164 (2000) 45-52.

[132] S. Vaynman, Z. Ying, F. Gomez-Pinilla, Hippocampal BDNF mediates the efficacy of exercise on synaptic plasticity and cognition, Eur. J. Neurosci. 20 (2004) 2580-2590.

[133] G.S. Griesbach, D.A. Hovda, R. Rolteni, A. Wu, F. Gomez-Pinilla, Voluntary exercise following traumatic brain injury: brain-derived neurotrophic factor upregulation and recovery of function, Neurosci. 125 (2004) 129-139.

[134] A. Wu, Z. Ying, F. Gomez-Pinilla, The interplay between oxidative stress and brain-derived neurotrophic factor modulates the outcome of a saturated fat diet on synaptic plasticity and cognition, Eur. J. Neurosci. 19 (2004) 1699-1707.

[135] F. Fumagalli, G. Racagni, M.A. Riva, The expanding role of BDNF: a therapeutic target for Alzheimer's disease?, Pharmacogenom. J. 6(1) (2006) 8-15. 